\title{
NC-6004, a novel cisplatin nanoparticle, in combination with pembrolizumab for head and neck cancer
}

\author{
Atsushi Osada* \\ NanoCarrier US LLC, 319 West Main St, Danville, VA, USA
}

After immune checkpoint blockades (ICBs) showed significant clinical results in a lot of oncology trials for several type of tumors, immune therapy became the fourth pillar of oncology treatment in addition to surgery, radiotherapy and chemotherapy including molecular targeting agents and treatment landscape was dramatically changed. However, chemotherapy still has an important role in cancer treatment and one of the widely used chemotherapeutic agents is cisplatin. Cisplatin is popularly used for the treatment of thoracic tumors, head and neck cancer, gastrointestinal cancers, gynecologic malignancies, osteosarcoma or malignant lymphoma but cisplatin treatment is associated with problematic neurotoxicity, renal toxicity restricting treatment duration or patient population due to comorbidities, e.g. renal impairment. Therefore, it provides tremendous clinical benefit if such toxicities could be improved. NC-6004 is a cisplatin nanoparticle developed using a cutting-edge micelle nanotechnology. Cisplatin is encapsulated into approximately $30 \mathrm{~nm}$ size polymeric micelles through the polymer-metal complex formation between polyethylene glycol poly (glutamic acid) block copolymers (PEG-P(Glu)) [1]. NC6004 can penetrate and accumulate into tumor lesion selectively by utilizing the phenomenon called enhanced permeability and retention (EPR) effect [2]. It is known that conventional cisplatin is excreted quickly from the human body but NC-6004 can circulate in blood stream longer period of time because of the outer PEG shell protects the micelle to being retained by the reticuloendothelial systems so that NC6004 releases cisplatin gradually by exchanging chloride ion in human body [1]. Therefore NC-6004 dramatically changes pharmacokinetic profile of conventional cisplatin meaning that NC-6004 increases AUC and decreases Cmax resulting in the better safety profile is expected compared to conventional cisplatin. Preclinical studies showed that NC-6004 demonstrated significantly lower nephrotoxicity and neurotoxicity compared to conventional cisplatin at an equivalent dose [3]. Besides the preclinical study showed that NC-6004 was preferably distributed to tumors contributing the better safety profile [3]. The FirstIn-Human (FIH) trial of NC-6004 was done in UK as monotherapy and the expected pharmacokinetic profile was observed [4]. So far eight clinical trials were conducted worldwide and some trials are still ongoing including pancreatic cancer phase III trial in Asia. In the FIH study the maximum tolerated dose (MTD) and the recommended phase II dose (RP2D) of NC-6004 monotherapy was determined to be $120 \mathrm{mg} / \mathrm{m}^{2}$ and $90 \mathrm{mg} / \mathrm{m}^{2}$, respectively [5]. Although a phase $1 \mathrm{~b} / 2$ trial conducted in the US was in combination with gemcitabine $1250 \mathrm{mg} /$ $\mathrm{m}^{2}$ which induces severe hematological toxicity the study reported that MTD and RP2D of NC-6004 were $135 \mathrm{mg} / \mathrm{m}^{2}$ which is higher than UK study result as well as popularly used cisplatin dose in daily practice [5]. This inconsistency can be explained by the difference of study design. UK phase I study had a 3+3 modified Fibonacci dose escalation design but the continuous reassessment method (CRM) was adopted in US phase 1b/II study [5]. Currently a new study of NC-6004 is being initiated for squamous cell carcinoma of head and neck (SCCHN) in combination with pembrolizumab. The study title is "Phase IIa/ IIb Clinical Trial of NC-6004 in Combination with Pembrolizumab in Subjects with Recurrent or Metastatic Squamous Cell Carcinoma of the Head and Neck Who Have Failed Platinum or a Platinum-containing Regimen" and the study is registered with ClinicalTrials.gov, number NCT03771820. This trial has two portions. The primary objective of the first portion is to determine optimal NC-6004 dose in combination with pembrolizumab. The second portion is head-to-head comparison of NC-6004 plus pembrolizumab vs pembrolizumab alone and the primary endpoint is PFS. This study is supported by five key elements observed from past NC-6004 clinical studies or currently available insights. The first observation is that there are some reports showing platinum compound induces oncologic immune modulation. It can be suggested that NC-6004 plus ICB combination could provide synergistic effect $[6,7]$. In addition to that, ICB plus platinum containing chemotherapy prolonged survival duration significantly compared to chemotherapy alone in Non-Small Cell Lung Cancer trials [8]. Those regimens were already approved in the US. The second rationale is relevant to why the target population is platinum pre-treated SCCHN patients. Platinum agents are rarely used for the population who previously have received platinum treatment in day-to-day medical practice. In NC-6004 US phase $1 \mathrm{~b} / \mathrm{II}$ study the tumor shrinkage was observed in 11 subject (55\%) and $67 \%$ of these subjects received prior platinum therapy. Two SCCHN subjects were enrolled in the study and those subjects had prior platinum therapy. Partial response was observed in a subject and another subject experienced tumor shrinkage within stable disease criteria. Besides those tumor shrinkages was maintained longer period of time. Therefore, it is expected that NC-6004 could be efficacious to platinum pre-treated SCCHN population. Another perspective is that nanoparticle compound could bypass the multi-drug resistance (MDR) [9]. P-glycoprotein (P-gp) is one of the most well-known ATP-biding cassette transporter acting to pump its substrates (toxins or drugs) out from inner cells to outside and it is said that approximately $50 \%$ of the anticancer drugs clinically available today including cisplatin are

*Correspondence to: Atsushi Osada, NanoCarrier US LLC, 319 West Main St, Danville, VA, 24541, USA, Tel: 781219 4958; E-mail: osada@nanocarrier.co.jp

Key words: immune checkpoint blockade, micelle, cisplatin, NC-6004

Received: April 29, 2019; Accepted: May 07, 2019; Published: May 10, 2019 
substrates of P-gp [9]. One of the mechanisms how to overcome the MDR by nanoparticle is that nanoparticle delivers high concentrated drugs released inside the tumor cell resulting in saturation of P-gp [9]. The third factor is the tolerability of the combinatorial drug. One of the big advantages of nanoparticle drug is its better safety profile owing to selective tumor accumulation as well as lower Cmax. In order to maximize such unique feature, it makes a lot of sense to combine with a good safety profile drug and ICB alone is definitely tolerable rather than chemotherapeutic agents or even some molecular targeting agents. The forth rationale is ICBs were approved in platinum pre-treated SCCHN worldwide already. The fifth justification is the dosing schedule of ICB. Currently pembrolizumab and nivolumab are approved for SCCHN. The administration schedule of pembrolizumab is every three weeks, on the other hand nivolumab's dosing schedule is every two weeks or every four weeks. NC-6004 was administered every three weeks so far, therefore pembrolizumab should be the best fit to NC-6004. The study is just initiating and First-Patient-In will be seen in the year of 2019. Apparently, the NC-6004 trial is a clinical study meaning that anticancer activity or safety profile is not warranted, however it is hoped that this trial could provide a valuable treatment opportunity to study subjects particularly who are in country where ICBs are not reimbursed or approved to SCCHN.

\section{Acknowledgment}

This study has received funding from "Orient Europharma Co., Ltd.

\section{References}

1. Nishiyama N, Okazaki S, Cabral H, Miyamoto M, Kato Y, et al. (2003) Novel cisplatinincorporated polymeric micelles can eradicate solid tumors in mice. Cancer Res 63 : 8977-8983. [Crossref]

2. Matsumura Y, Maeda H (1986) A new concept for macromolecular therapeutics in cancer chemotherapy: mechanism of tumoritropic accumulation of proteins and the antitumor agent smancs. Cancer Res 46: 6387-6392. [Crossref]

3. Uchino H, Matsumura Y, Negishi T, Koizumi F, Hayashi T, et al. (2005) Cisplatinincorporating polymeric micelles (NC-6004) can reduce nephrotoxicity and neurotoxicity of cisplatin in rats. Br J Cancer 93: 678-687. [Crossref]

4. Plummer R, Wilson RH, Calvert H, Boddy AV, Griffin M, et al. (2011) A phase I clinical study of cisplatin-incorporated polymeric micelles (NC-6004) in patients with solid tumours. Br J Cancer 104: 593-598. [Crossref]

5. Subbiah V, Grilley-Olson JE, Combest AJ, Sharma N, Tran RH, et al. (2018) Phase Ib/II Trial of NC-6004 (Nanoparticle Cisplatin) Plus Gemcitabine in Patients with Advanced Solid Tumors. Clin Cancer Res 24: 43-51. [Crossref]

6. Patel MA, Kim JE, Ruzevick J, Lim M (2015) Present and future of immune checkpoint blockade: Monotherapy to adjuvant approaches. World J Immunol 5: 1-15.

7. de Biasi AR, Villena-Vargas J, Adusumilli PS (2014) Cisplatin-induced antitumor immunomodulation: a review of preclinical and clinical evidence. Clin Cancer Res 20: 5384-5391. [Crossref]

8. Gandhi L, Rodriguez-Abreu D, Gadeel S, Tafreshi A, Gümüş M et al. (2018) Pembrolizumab plus chemotherapy in metastatic non-small-cell lung cancer. $N$ Engl $J$ Med 378: 2078-2092. [Crossref]

9. Dong X, Mumper RJ (2010) Nanomedicinal strategies to treat multidrug-resistant tumors: current progress. Nanomedicine (Lond) 5: 597-615. [Crossref]

Copyright: (C2019 Osada A. This is an open-access article distributed under the terms of the Creative Commons Attribution License, which permits unrestricted use, distribution, and reproduction in any medium, provided the original author and source are credited. 